\title{
Management of idiopathic intracranial hypertension with repeated lumboperitoneal shunt malfunction
}

\author{
Hesham Atef Elshitany ${ }^{*}$ (D) and Ehab Abd ELHaleem Ali
}

\begin{abstract}
Introduction: The incidence of idiopathic intracranial hypertension is $1 / 100,000$ population per year. The widely accepted surgical treatment for idiopathic intracranial hypertension $(I I H)$ in the last four decades has been the insertion of lumboperitoneal (LP) shunt (Greer, Clin Neurosurg 15:161-174, 1968; Weisberg, Medicine 54:197-207, 1975). The LP shunt is associated with high revision rates.
\end{abstract}

Patients and methods: This is a prospective study that was done between 1 January 2014 and 31 December 2014, including seven patients with $1 \mathrm{H}$ and a lumboperitoneal shunt malfunction. These patients were treated by an insertion of a ventriculoperitoneal (VP shunt). The Medtronic medium pressure shunt was used. The patients were followed up at 1, 3, 6, and 12 months.

Results: In four patients, the shunt malfunction was due to slippage of the peritoneal end either into subcutaneous fat of the abdomen in three patients or into subcutaneous fat of the back (with occurrence of shunt infection) in one patient. In one patient, the failure was due to the slippage of both tubes into the subcutaneous abdominal layer, while in two patients, shunt failure was due to obstruction with adequate shunt position.

All patients were females (100\%). Age ranged from 22 to 48 years with mean age at 32 years. Five patients (71\%) had previous one LP shunt revision surgery while two patients (29\%) had previous two revision surgeries. Six patients (85\%) showed elevated ICP on CSF manometry, and one patient with wound collection, who showed evident infection, was enrolled in the algorithm for positive infection. Brain imaging showed slit ventricles in five patients and near normal ventricles in two patients. All patients experienced smooth postoperative recovery. Postoperative images were satisfactory regarding position of distal and proximal shunt tubes. Headache was improved in all patients within the first postoperative day, while visual symptoms improved markedly within 2 weeks postoperatively.

Conclusion: The use of VP shunts is extremely beneficial in the treatment of IIH-associated LP shunt malfunction especially with the new technologies in the placement of the ventricular end, and conducting a multicenter trial is recommended to re-evaluate if the primary surgical treatment of IIH will continue to be the LP shunts or shift to the use of VP shunts.

Keywords: Idiopathic intracranial hypertension, Shunt malfunction, VP shunt

\footnotetext{
* Correspondence: helshitany@gmail.com

Neurosurgery Department, Faculty of Medicine, Cairo University, 5 Alsaraya

Street, El-Manial, Cairo, Egypt
} 


\section{Introduction}

The incidence of idiopathic intracranial hypertension is $1 /$ 100,000 population per year or $19.3 / 100000$ in obese women with age between 20 to 44 years [1]. The only major morbidity associated with IIH is the visual troubles which occur in most of the patients [2]. Idiopathic intracranial hypertension (IIH) is characterized by signs and symptoms of increased intracranial pressure in a conscious patient. In these patients, the neuroimaging and CSF analysis are normal. Lumbar puncture and CSF manometry show increased intracranial pressure. It is usually diagnosed according to modified Dandy criteria for IIH [3].

The widely accepted surgical treatment for IIH in the last four decades has been the insertion of LP shunt $[4,5]$. The LP shunt is associated with high revision rates [6-8]. The ventriculoperitoneal (VP) or the ventriculoatrial (VA) shunts have been used less frequently in these patients. This is due to the difficulty in inserting the ventricular end into a non-dilated or a slit ventricle [7, 9-17]. To our knowledge, there is no reported comparison between the LP and the VP shunts for the treatment of the IIH.

\section{Patients and methods}

This is a prospective study that was conducted in the Neurosurgery Department of Cairo University Hospitals between the 1 January 2014 and 31 December 2014. All patients were presented with IIH and LP shunts malfunction with history of previous LP shunt revision. These patients were at least operated upon twice, the first was for shunt insertion while the second was for shunt revision. Each patient was subjected to the following: plain $\mathrm{x}$-ray film (postero-anterior and lateral views), CT brain, MRI brain, fundus examination, visual perimetry, and CSF pressure measurement and analysis through a lumbar tap to confirm shunt malfunction (obstruction or infection). Management of the cases was done according to the following algorithm in Fig. 1.

In this series, seven patients were treated. Two patients had shunt obstruction (recurrence of symptoms of benign intracranial hypertension, although having adequate shunt position). Four patients showed migration of the peritoneal catheter with adequate position of proximal intrathecal catheter. Three of them had slippage of distal end in the subcutaneous fat of the abdomen while one patient presented with slippage of the distal end in the subcutaneous fat of the back (back wound collection with local signs of infection) and shunt infection. One patient had slippage of both tubes in subcutaneous fat of the abdomen.

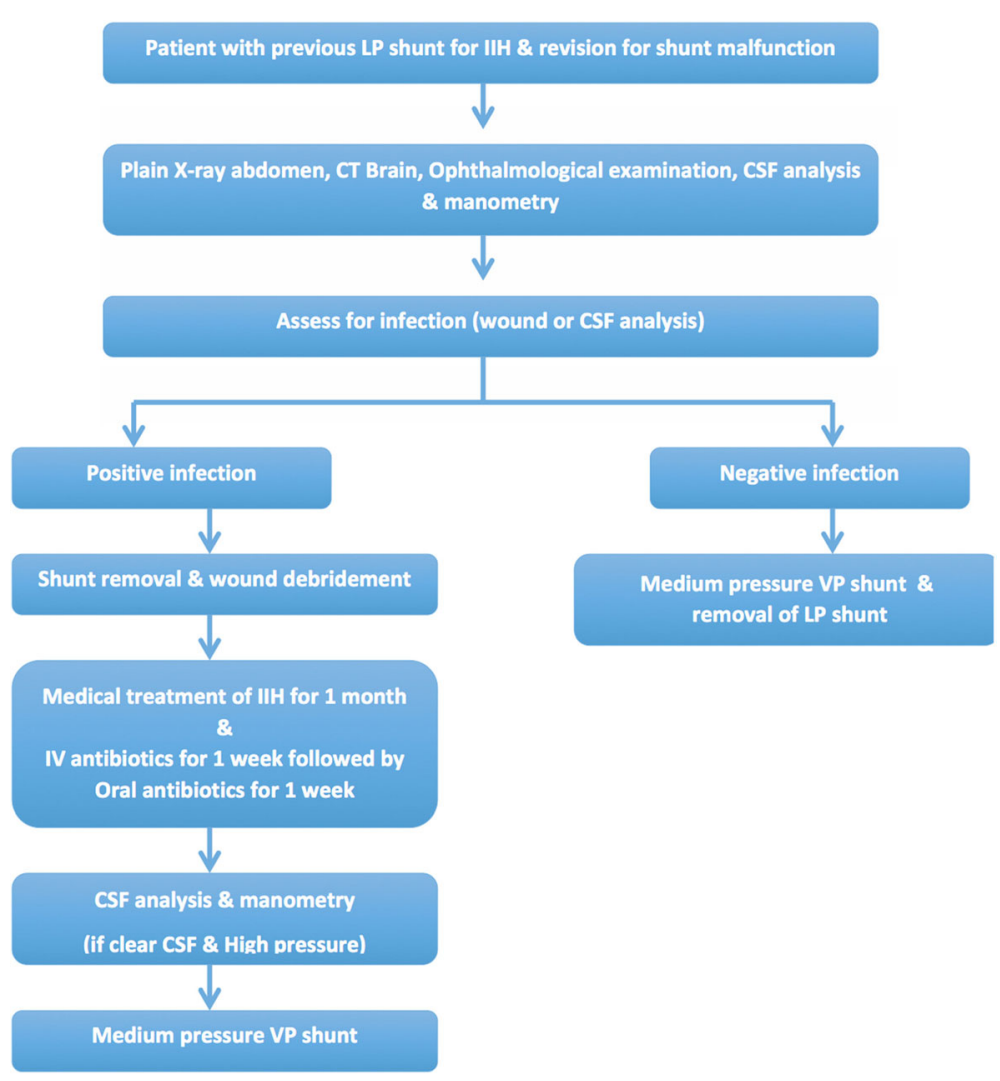

Fig. 1 Algorithm for treatment of failed LP cases with IH 
The patient, with backward migrated distal tube and shunt infection, presented with back wound collection, macerated wound edges, redness, and localized tenderness. Percutaneous aspiration of subcutaneous collection and cytological examination were done. It showed leukocytosis with neutrophils predominance and elevated proteins (Fig. 1), and culture and sensitivity results revealed Staphylococcus aureus. Surgical debridement of the wound and shunt removal were done. Intravenous vancomycin and amikacin were given for 1 week followed by oral antibiotics in the form of linezolid and levofloxacin for another 2 weeks. One month later, lumbar tap for CSF sample and analysis revealed acellular CSF with absent signs of wound infection. The patient was operated upon by $\mathrm{V} / \mathrm{P}$ shunt.

All seven patients were put in supine position. The primary challenge has been the ability to cannulate normal or smaller than normal size ventricles in patient with IIH. A C-shaped incision over laying the Kocher's burr hole $(2$ to $3 \mathrm{~cm}$ lateral to mid line and $1 \mathrm{~cm}$ anterior to coronal suture) was used. The abdominal incision was made in the mid line between the umbilicus and sternum or in a subcostal muscle splitting incision. In both incisions, the new abdominal incision was away from the previous $\mathrm{L} / \mathrm{P}$ one.

The Medtronic medium pressure shunt was used.

The distal catheter was tunneled subcutaneously (often using an intervening incision). The distal end was connected to the reservoir. The proximal end was inserted aiming towards the ipsilateral inner canthus in coronal plane and towards external auditory meatus in antero-posterior plan. It was inserted (free-handed) for about 5 to $6 \mathrm{~cm}$.

Once the proximal catheter is in place, it is connected to the other side of the valve and the valve is fixed in place.

The non-functioning LP shunt was removed at the end of the procedure, and a blood patch was injected in place deep to the back muscles.

Postoperative CT brain (Fig. 2) and plain abdominal and pelvic $\mathrm{x}$-ray were done to ensure the perfect position on the second postoperative day.

The patients were followed up at 1, 3, 6, and 12 months. The skin wounds were examined for healing. The filling and emptying time of the reservoir was noted. Postoperative CT brain and plain abdominal and pelvic X-ray were done. Visual assessment was done in the form of an assessment of visual acuity, visual field, and fundus examination.

A definition of shunt failure was based on the return of the preoperative symptoms and high CSF opening pressure.

\section{Results}

Seven patients were presented with IIH and lumboperitoneal shunt malfunction. These patients were treated by insertion of ventriculoperitoneal (VP shunt).
All patients were female (100\%). Age ranged from 22 to 48 years with mean age at 32 years.

Five patients (71\%) had previous one LP shunt revision surgery while two patients $(29 \%)$ had previous two revision surgeries.

The malfunction was diagnosed by recurrence of symptoms (headache or visual symptoms) or presence of collection at the site of the wound.

Five patients $(71 \%)$ were presented with shunt failure due to tube migration (one of them had wound and shunt infection), while two patients (29\%) presented with shunt failure due to shunt obstruction with proper shunt position.

Six patients (85\%) showed elevated ICP on CSF manometry, and one patient with wound collection, who showed evident infection, was enrolled in the algorithm for positive infection.

Brain imaging showed slit ventricles in five patients and near normal ventricles in two patients.

All patients experienced smooth postoperative recovery. Postoperative images were satisfactory regarding position of distal and proximal shunt tubes.

Headache was improved in all patients within the first postoperative day, while visual symptoms improved markedly within 2 weeks postoperatively.

Postoperative CT brain and plain abdominal and pelvic $\mathrm{x}$-ray revealed proper shunt position in all patients.

\section{Follow-up}

Visual assessment (visual acuity, visual field, and fundus examination) and clinical examination of shunt integrity through appropriate filling and emptying time of shunt reservoir and perfect healing of cranial and abdominal wounds of the shunt were followed up postoperatively at intervals of $1,3,6$, and 12 months.

\section{Discussion}

Pseudotumor cerebri or idiopathic intracranial hypertension (IIH) is a syndrome of unclear etiology characterized by increased intracranial pressure. The modified Dandy criteria (Table 1) are used to diagnose $\mathrm{IIH}$ and to rule out other conditions that are similar to $\mathrm{IIH}$ and may need different treatment [18].

Surgery is indicated for noncompliant or intolerant patients on the medical treatment or patients not responding to medical treatment.

There are several surgeries that can be done to treat IIH but the most commonly used are the CSF shunting procedures. The LP shunts, because of morbid obesity, have a lot of complications, either slippage or obstruction. As for the VP shunts, the placement of the ventricular catheter is very difficult because of the small ventricular size. The new advanced technologies in the placement of the ventricular end using neuroendoscopy 


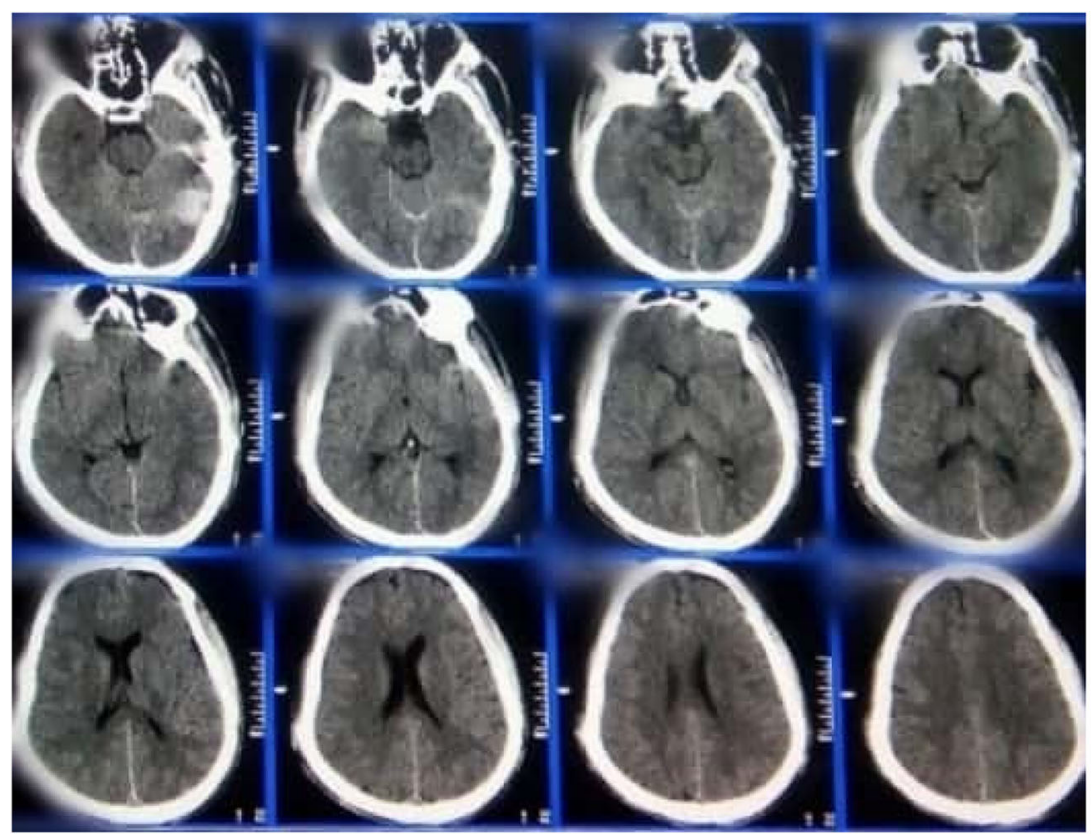

a

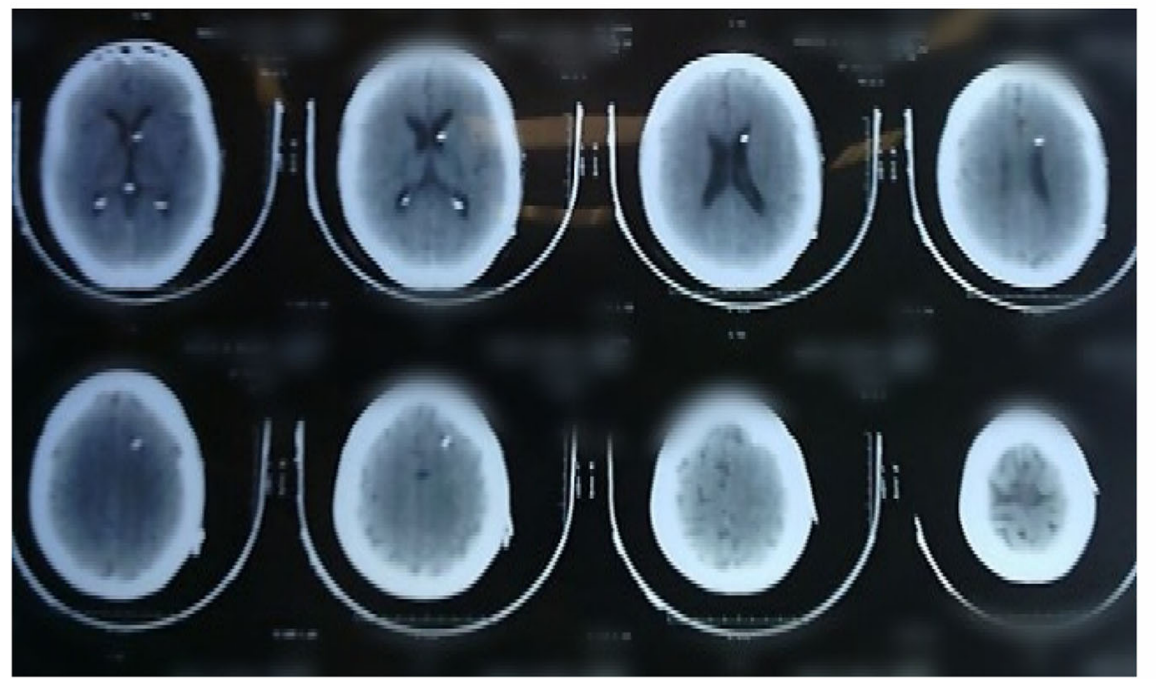

b

Fig. 2 CT brain pre (a) and post (b)

Table 1 Modified Dandy criteria

1. Symptoms and signs of raised intracranial pressure.
2. No localizing signs in the neurological examination (except
abducens nerve palsy).
3. Normal neuroimaging (except empty sella).
4. Opening pressure of lumbar puncture of greater than $250 \mathrm{~mm}$
water, with normal CSF
5. Alert and awake patient
6. No other cause of raised intracranial pressure

or neuronavigation make the ventriculoperitoneal shunt a promising alternative treatment for IIH [19]. This would help to avoid complications of LP shunts including migration or obstruction [20].

A series of 17 patients treated with VP shunts for IIH were reported by Bynke et al. and with 1.8-12.8 years (mean, 6.5 years) follow-up. All the clinical manifestations of IIH were effectively treated by VP shunt. In this study, seven patients were operated upon by one or two (a total of nine) surgical revisions [21].

A series of 21 patients treated with 32 ventricular shunting procedures $(20 \mathrm{VP}, 10 \mathrm{VA}$, and 2 ventriculopleural) for IIH was reported by Woodworth et al. All the clinical manifestations of IIH especially the headache 
improved after the placement of the shunt. The ventricular shunt failure rates were $10 \%, 20 \%, 50 \%$, and $60 \%$ after $3,6,12$, and 24 months respectively post shunt insertion. Of the revisions, $67 \%$ were done due to distal obstruction, $20 \%$ were due to over drainage, and $6.5 \%$ were due to CSF leak or distal catheter migration. There were no shunt infection or proximal catheter obstruction [22].

The efficacy of CSF diversion in the treatment of idiopathic intracranial hypertension was reviewed from six institutions by Rosenberg et al. in a series of 37 patients treated with $10 \mathrm{VP}$ shunts and $73 \mathrm{LP}$ shunts. Only 14 patients did not need a revision of their shunts. The average time between the first operation and the revision was 9 months. The most common indications for shunt revision were either shunt failure (55\%) or low-pressure headaches (21\%). Postoperatively, most of the patient's clinical manifestations, especially the vision, stabilized or improved postoperatively. However, postoperative deterioration of vision was seen in six patients, complete loss of vision despite adequate shunt function was seen in four patients, loss of vision after initial improvement was seen in three patients, and improvement of vision in one eye and deterioration of vision in the other eye was seen in two patients. The relapse of IIH symptoms after shunt malfunction can occur as late as 7 years from the initial shunt procedure. In conclusion, the shunt procedures have a high frequency of side effects and high failure rates [16].

The records of all shunts placed between 1973 and 2003 for IIH at one institution were reviewed by McGirt et al. in a series of 42 patients treated with $36 \mathrm{VP} / \mathrm{VA}$ shunts and 79 LP shunts. All the clinical manifestations of IIH especially the headache were significantly improved after the placement of the shunt in $95 \%$ of the cases. The recurrence of a severe headache occurred in $19 \%$ and $48 \%$ after 12 and 36 months, respectively, post shunt insertion despite a well-functioning shunt. The LP shunts had a threefold increase in shunt obstruction in comparison to VP and VA shunts. However, the risk of distal migration, over drainage and shunt infection was the same in both the LP shunt and the VP/VA shunt groups. In conclusion, shunts are extremely beneficial in the acute treatment of $\mathrm{IIH}$-associated intractable headache, and the majority of patients had a long-term relief. Treatment of IIH with VP/VA shunts was associated with a decreased incidence of shunt obstruction as compared to LP shunts [23].

In our study, a series of seven patients presented with LP shunt malfunction, four of the patients showed a failure of the peritoneal catheter while one patient showed a failure of both proximal and peritoneal catheter and two patients showed perfect shunt position. All the patients showed elevated ICP on CSF manometry except one patient with wound collection. On fundus examination, four patients showed papilledema while three patients showed pale optic discs. All the clinical manifestations of IIH especially the headache and visual symptoms were significantly improved after the revision procedure.

Most of the studies investigating the efficacy of different treatment modalities for IIH are single-center retrospective studies with relatively small numbers of patients, which aim to share the experience of individual centers treating patients with IIH. Our study also falls into this category except that it was a prospective study. This clearly highlights the need for large multicenter trials to determine the best treatment option for these patients.

\section{Conclusion}

The use of VP shunts is extremely beneficial in the treatment of IIH-associated LP shunt malfunction especially with the new technologies in the placement of the ventricular end, and conducting a multicenter trial is recommended to re-evaluate if the primary surgical treatment of IIH will continue to be the LP shunts or shift to the use of VP shunts (either free-handed strictly following the classic anatomical facts, as in our study, or with the use of neuronavigators in the placement of the ventricular end).

\section{Acknowledgements \\ Mohamed Amin Roshdy, Assistant Lecturer of Neurosurgery, Cairo University. \\ Funding \\ Self funding. \\ Availability of data and materials \\ The dataset supporting the conclusions of this article is included within the article.}

Authors' contributions

HE contributed in the design, data collection, manuscript drafting, and surgical procedures. EAEH contributed in the revision of the intellectual content, data analysis, follow-up, and surgical procedures. All authors read and approved the final manuscript.

Ethics approval and consent to participate

- Ethical Committee, Department of Neurosurgery, Cairo University

- The committee's reference number:

Dr. Mostafa Kotb $(+2012) 22118951$

Professor of Neurosurgery

Head of Ethical Committee, Department of Neurosurgery,

Cairo University

- Date of approval: 2/1/2014

- All participants provided informed consent to participate in the study.

Consent for publication

All participants provided informed written consent for publication of their data included within the study.

Competing interests

The authors declare that they have no competing interests. 


\section{Publisher's Note}

Springer Nature remains neutral with regard to jurisdictional claims in published maps and institutional affiliations.

Received: 2 August 2017 Accepted: 17 December 2018

Published online: 09 January 2019

\section{References}

1. Durcan FJ, Corbett JJ, Wall M. The incidence of pseudotumor cerebri: population studies in lowa and Louisiana. Arch Neurol. 1988;45(8):875-7.

2. Wall M, George D. Idiopathic intracranial hypertension: a prospective study of 50 patients. Brain. 1991;114:155-80

3. Smith JL. Whence pseudotumor cerebri? J Clin Neuroophthalmol. 1985:5(1):55-6.

4. Greer M. Management of benign intracranial hypertension (pseudotumor cerebri). Clin Neurosurg. 1968:15:161-74.

5. Weisberg LA. Benign intracranial hypertension. Medicine. 1975;54:197-207.

6. Burgett RA, Purvin VA, Kawasaki A. Lumboperitoneal shunting for pseudotumor cerebri. Neurology. 1997;49:734-9.

7. Eggenberger ER, Miller NR, Vitale S. Lumboperitoneal shunt for the treatment of pseudotumor cerebri. Neurology. 1996:46:1524-30.

8. Kang S. Efficacy of lumbo-peritoneal versus ventriculo-peritoneal shunting for management of chronic hydrocephalus following aneurysmal subarachnoid haemorrhage. Acta Neurochir. 2000;142:45-9.

9. Amacher AL, Spence JD. Spectrum of benign intracranial hypertension in children and adolescents. Childs Nerv Syst. 1985;1:81-6.

10. Beatty RA. Cervical-peritoneal shunt in the treatment of pseudotumor cerebri. Technical note. J Neurosurg. 1982;57:853-5.

11. Bulens C, De Vries WA, Van Crevel H. Benign intracranial hypertension. A retrospective and follow-up study. J Neurol Sci. 1979;40:147-57.

12. Johnston I, Besser M, Morgan MK. Cerebrospinal fluid diversion in the treatment of benign intracranial hypertension. J Neurosurg. 1988;69:195-202.

13. Johnston I, Paterson A. Benign intracranial hypertension. I Diagnosis and prognosis. Brain. 1974;97:289-300.

14. Johnston I, Paterson A. Benign intracranial hypertension. II CSF pressure and circulation. Brain. 1974;97:301-12

15. Meador KJ, Swift TR. Tinnitus from intracranial hypertension. Neurology. 1984;34:1258-61.

16. Rosenberg ML, Corbett JJ, Smith C, et al. Cerebrospinal fluid diversion procedures in pseudotumor cerebri. Neurology. 1993:43:1071-2.

17. Vander Ark GD, Kempe LG, Smith DR. Pseudotumor cerebri treated with lumbar-peritoneal shunt. JAMA. 1971;217:1832-4.

18. Dandy WE. Intracranial pressure without brain tumor - diagnosis and treatment. Ann Surg. 1937;106(4):492-513.

19. Tulipan N, Lavin PJ, Copeland M. Stereotactic ventriculoperitoneal shunt for idiopathic intracranial hypertension: technical note. Neurosurgery. 1998;43:175-7.

20. Wong JM, Ziewacz JE, Ho AL, Panchmatia JR, Bader AM, Garton HJ, et al. Patterns in neurosurgical adverse events: cerebrospinal fluid shunt surgery. Neurosurg Focus. 2012;33(5):E13.

21. Bynke G, Zemack G, Bynke H, Romner B. Ventriculoperitoneal shunting for idiopathic intracranial hypertension. Neurology. 2004;63(7):1314-6.

22. Woodworth GF, McGirt MJ, Elfert P, Sciubba DM, Rigamonti D. Frameless stereotactic ventricular shunt placement for idiopathic intracranial hypertension. Stereotact Funct Neurosurg. 2005;83(1):12-6.

23. McGirt MJ, Woodworth G, Thomas G, Miller N, Williams M, Rigamonti D. Cerebrospinal fluid shunt placement for pseudotumor cerebri-associated intractable headache: predictors of treatment response and an analysis of long-term outcomes. J Neurosurg. 2004;101(4):627-32.

\section{Submit your manuscript to a SpringerOpen ${ }^{\circ}$ journal and benefit from:}

- Convenient online submission

- Rigorous peer review

- Open access: articles freely available online

- High visibility within the field

- Retaining the copyright to your article

Submit your next manuscript at $\boldsymbol{\nabla}$ springeropen.com 